\title{
Functional outcome of arthroscopic meniscal repair in meniscal tears: a prospective study in tertiary care hospital in North India
}

\author{
Simran Preet Singh, Manish Singh, Pankaj Vir Singh*, Sumitpal Singh, Mudasir Arif
}

Department of Orthopaedics, GMC, Jammu, Jammu and Kashmir, India

Received: 15 April 2021

Accepted: 12 May 2021

\section{*Correspondence:}

Dr. Pankaj Vir Singh,

E-mail: pankajcoolpal@gmail.com

Copyright: ( $)$ the author(s), publisher and licensee Medip Academy. This is an open-access article distributed under the terms of the Creative Commons Attribution Non-Commercial License, which permits unrestricted non-commercial use, distribution, and reproduction in any medium, provided the original work is properly cited.

\begin{abstract}
Background: A Meniscal injury can result in intra articular damage and which can eventually result in osteoarthritis if not treated on time and the objective should be to save the meniscus wherever possible. This study was done to analyse the functional outcome of arthroscopic meniscal repair done in different types of Meniscal tears.

Methods: The study was conducted on a group of 28 patients with meniscal injury admitted in government medical college Jammu from November 2018 to October 2020. All the tears were located in the red-red and red-white zones. The functional outcome was assessed on the basis of Lysholm Tegner scoring. A standard rehabilitation protocol was followed post repair.

Results: Twenty-eight patients were included in the study. Male patients outnumbered the females and the incidence of injury was more on the right side. Sports injuries were the most common mode of injury, followed by road traffic accidents and falls. Bucket handle tears were most commonly seen in the group of patients. ACL injury was the major associated injury to be noted. The functional outcome between pre and post operation was statistically significant $(\mathrm{p}<0.05)$. In one of the patient failures was seen. Two patients were lost to follow up and were not included in the study. Conclusions: Arthroscopic meniscal repair is a has a very good functional outcome according to our study and should be preferred over meniscectomy (subtotal or total), as meniscus being a very important shock absorber present between the knee joint can lead to delay in osteoarthritis of knee joint. Therefore, our priority should be to save the meniscus and restore the normal anatomy of the knee joint for its biomechanical stability and normal function.
\end{abstract}

Keywords: Meniscal repair, Sports injuries, Knee arthroscopy

\section{INTRODUCTION}

The knee is one of the most frequently injured joints because of its anatomical structure, its exposure to external forces and the functional demands placed on it. Menisci transmit approximately $50 \%$ of weight bearing forces across the knee in extension and $85-90 \%$ in flexion. ${ }^{1}$ The menisci act as a joint filler and shock absorber, compensating gross abnormality between femoral and tibial articular surfaces. The menisci are believed to have joint lubrication function, helping to spread synovial fluid throughout the joint and aiding the nutrition to articular cartilage. $^{2-4}$
The menisci, once thought as a vestige of leg muscle, have been considered as a vital structure with various functions: chondroprotection through weight distribution by enlarging the contact area between the femur and the tibia. ${ }^{5}$ Long-term follow-up studies showed that virtually all meniscectomized knees develop arthritic changes with time. ${ }^{6}$ Absence of the menisci is associated with joint instability and to increased cartilage degeneration. ${ }^{7}$ Therefore, meniscal repair should be preferable to meniscectomy. Medial meniscal tears occur more frequently than tears of the lateral meniscus, at a ratio of approximately $2: 1 .^{8}$ 
The goals of surgical intervention for meniscal tears are to relieve pain, facilitate pre-injury level daily living activities, and prevent early degeneration of the knee joint.

\section{METHODS}

A Prospective cohort study was conducted in our institute after seeking approval from IEC. About 30 patients with suspected Meniscal injury admitted in govt medical college Jammu will be included in study after obtaining informed consent from. A detailed history, knee examination and MRI will be done. The three tests will be performed pre anaesthesia results reported. The patients undergo arthroscopic evaluation, intra op findings were recorded in recorder and Meniscal repair done. There postoperative results were then compared with both sets of patients. Two patients were lost in follow up and thus were excluded from study. One of the patient's failure was recorded.

Patients in age group 18-60 years with suspected isolated meniscal tear such as longitudinal, horizontal, radial, oblique, bucket handle. Patients belonging to the age group of 18-60 years with Meniscal Tear with associated ACL injury, medial collateral ligament injuries, lateral collateral ligament injury and Chondral injuries were included in the study. Exclusion criteria consisted of patients with ACL reinjuries, associated periarticular fracture, associated ipsilateral lower limb fractures, with posterior cruciate Ligament (PCL) injury and patients having complex meniscal tears.

All cases were performed under regional anaesthesia (Spinal) with the patient in supine position. Intravenous antibiotic prophylaxis was given. Diagnostic arthroscopy was performed to assess injuries. Tourniquet was used in all the cases (Figure 3). Outside in sutures for anterior horn tears, radial tears, reduction of bucket handle tears. Inside out sutures used for middle third tears, peripheral capsular. All inside technique was used for posterior horn tears.

Standard rehabilitation protocol was followed postsurgery. Gentle knee physiotherapy was started after $1^{\text {st }}$ week of surgery and during subsequent weeks range of motion was increased to around 90 degrees at the end of $1^{\text {st }}$ month. Open chain exercises were beginning at 6 weeks followed by wall slides and lunges.

\section{RESULTS}

Pre-operative assessment was done using Mc Murrays test, Medial joint line tenderness, x-ray knee, MRI knee, and Lysholm-Tegner score. Functional assessment of patients was done post operatively with Lysholm-Tegner score. The scores of both preoperative and post-operative assessments were expressed as mean \pm SD. The normality of the data was examined using appropriate tests (Shapiro Wilk test) and the scores were compared between pre-and post-operative time points using tests (unpaired t test and Fisher exact test). All statistical analysis were considered significant at $p<0.05$ level of significance. Software used for statistical analysis was Epi-info version 3.0 and SPSS. Total number of patients at the beginning of our study was 30, 2 patients were lost to follow up and 1 had failed Repair.28 patients completed the study. Patients ranged from 20-54 years in the group with an average age of 31.46 .

Majority of the patients in the study were males (69\%). Right side injury (54\%) was more commonly seen. Time since injury ranged from $0.5-10$ months with a mean of $4.5 \pm 2.6$ months. The most common mode of injury in our study was sports injury with 13 patients $(46.4 \%)$, followed by RTA with 9 patients $(32.1 \%)$, fall accounted for 4 patients (14\%) and direct blow 2 patients (7\%) (Figure 1). 14 patients $(50 \%)$ had a bucket handle tear. Longitudinal tear was present in 6 patients $(23 \%) .4$ patients $(14 \%)$ had a horizontal tear. 3 patients $(12 \%)$ presented with a radial tear and 1 had an oblique tear (Figure 2).

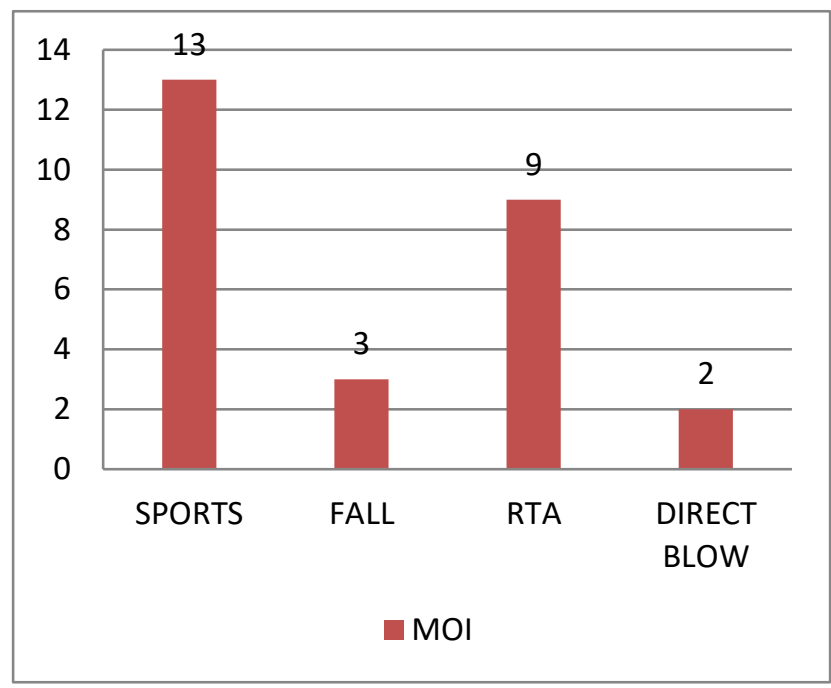

Figure 1: Bar graph of mode of injury distribution.

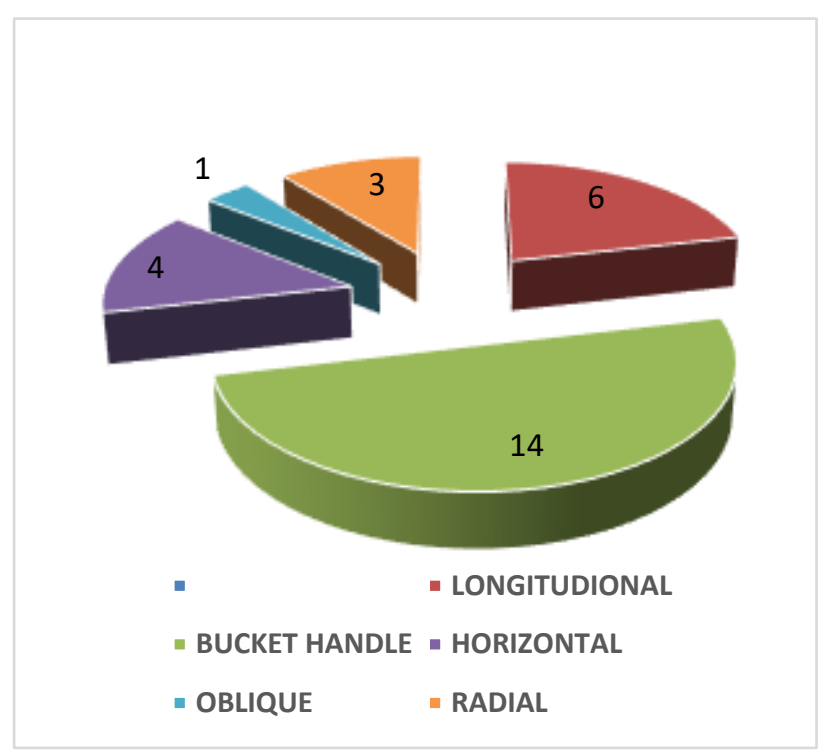

Figure 2: Pie chart of type of meniscal tears. 
Among 28 patients 14 had associated ACL injury (50\%), medial collateral ligament $1(3.5 \%)$, chondral injury 2 $(7 \%)$ and rest had isolated medial meniscus $9(32.3 \%)$ and isolated lateral meniscus was seen in $2(7 \%)$ patients. Complications recorded among 28 patients, 3 had numbness (12\%), 1 had swelling (4\%), 1 had effusion $(4 \%), 2$ had pain $(8 \%)$ and 0 had local infection $(0 \%)$.

Lysholm Tegner scoring done at the end of 12 months showed, 12 patients had excellent results, 15 had a good outcome followed by 1 patient who had a poor outcome. The mean Lysholm Tegner score was $88.42 \pm 9.1$ at the final follow up.

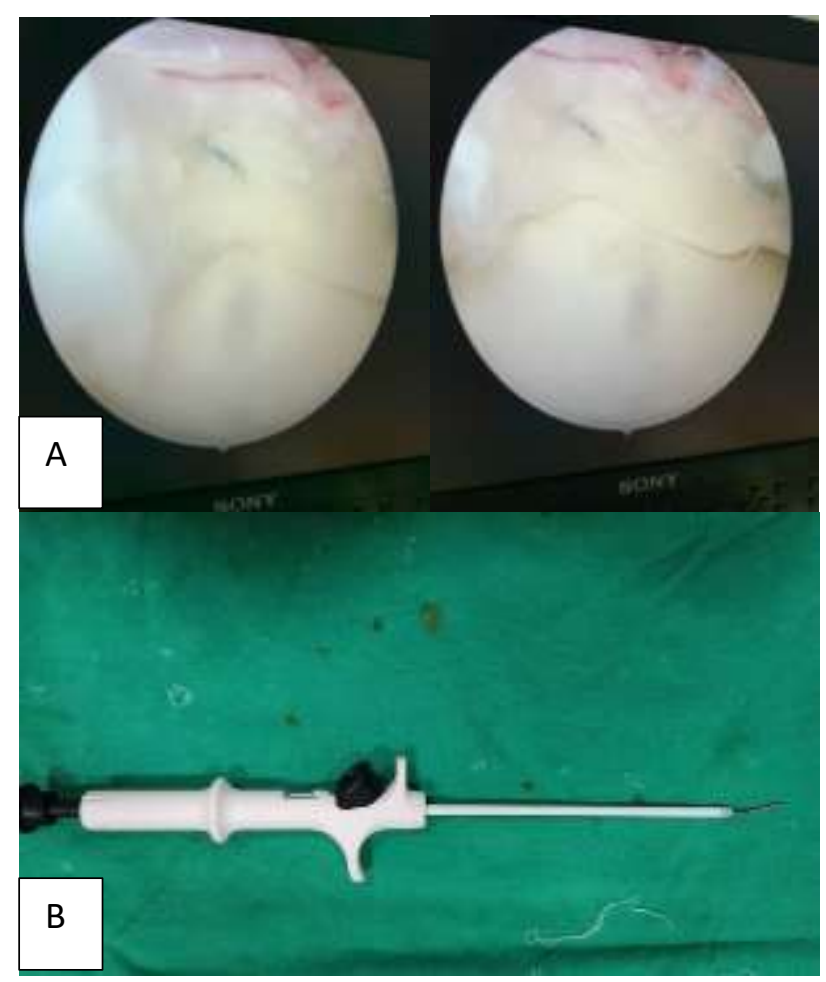

Figure 3: (A): All inside repair and (B): Fast-fix device.

\section{DISCUSSION}

Our study was done in government medical college Jammu from November 2018 to October 2020. 30 patients were included at the beginning of the study, 2 patients were lost to follow up and finally 28 patients completed the study. Mean time between injury and surgery was $4.5 \pm 2.6$ months. Different authors have had varying results on the time between injury and surgery. In a study by Deveci et al, mean time between injury and surgery was 8.7 weeks. ${ }^{9}$ The average age of patients included in the study was 31.46 years. Choi et al conducted a similar study with a mean age of 27.7 years and in a study by Stein et al mean age was 31.5 years. ${ }^{10,11}$ The most common mode of injury was sports injury $(46.4 \%)$ followed by RTA in $9(32.1 \%)$, fall in $4(14 \%)$ and direct blow in $2(7 \%)$ patients.
Right knee was found to be involved more than left knee. In the study by Lutz et al $53 \%$ patients had a right knee involvement. ${ }^{12}$ Fourteen $(50 \%)$ out of the 28 patients had associated ACL injury, $9(32.3 \%)$ patients had isolated medial meniscal injuries, $2(7 \%)$ patients had an isolated lateral meniscal injury, $1(3.5 \%)$ had medial meniscus with medial collateral injury, pure chondral lesion was seen in $2(7 \%)$ patients. Barret et al concluded that associated ACL injuries $(53.8 \%)$ were seen more frequently. ${ }^{13}$ Kimura et al in his study showed that ACL injury was associated in $71.4 \%$ of the total patients. ${ }^{14}$ In a study by Kocabey et al $59.5 \%$ patients had an associated ACL injury. ${ }^{15}$ Haas et al in his study showed $58.5 \%$ patients had a concomitant ACL injury. ${ }^{19}$ Complications such as numbness was seen in $3(10.5 \%)$ patients, $2(7 \%)$ had pain and $1(3.5 \%)$ had effusion. No infections were reported. Austin et al in his study reported numbness in $7 \%$ patients, effusion in $2 \%$ and infection in $1 \%$ patients. $^{16}$ Stone described neuropraxia in $28 \%$ patients. ${ }^{17}$

Lysholm Tegner score at final follow up was 88.42 \pm 9 . Stein et al, in his study reported mean LT score of $91.54 \pm 8.95$ at final follow up. Albertoni et al found out the mean LT score of $89.95 \pm 10.39$ at the end of his study (Table 1). ${ }^{18}$

Table 1: Comparison of Lysholm Tegner scoring outcome in various studies.

\begin{tabular}{|llll|}
\hline Study & Year & $\begin{array}{l}\text { Total } \\
\text { patients }\end{array}$ & $\begin{array}{l}\text { Lysholm-Tegner (at } \\
\text { final follow up) }\end{array}$ \\
\hline $\begin{array}{l}\text { Our } \\
\text { study }\end{array}$ & 2021 & 28 & $88.42 \pm 9.1$ \\
\hline $\begin{array}{l}\text { Hass } \\
\text { et al }\end{array}$ & 2005 & 37 & $93.73 \pm 6.58$ \\
\hline $\begin{array}{l}\text { Stein } \\
\text { et al } \mathbf{l}^{\mathbf{1 1}}\end{array}$ & 2010 & 26 & $91.54 \pm 8.95$ \\
\hline $\begin{array}{l}\text { Uzun } \\
\text { et al }\end{array}$ & 2019 & 184 & $88.4 \pm 11.7$ \\
\hline $\begin{array}{l}\text { Albertoni } \\
\text { et al }\end{array}$ & 2013 & 21 & $89.95 \pm 10.39$ \\
\hline
\end{tabular}

\section{CONCLUSION}

This study was carried out prospectively on 28 patients. Male patients outnumbered the females. There was higher incidence of injury on the right-side sports injuries were the most common mode of injury, followed by road traffic accidents and falls. Twelve patients had excellent results with Lysholm-Tegner score $>90$ with a mean average of $88.42 \pm 9.1$, compared to $49.73 \pm 4.3$ at presentation which was found to be statistically significant.

We can conclude that arthroscopic Meniscal repair is a has a very good functional outcome according to our study and should be preferred our meniscectomy (subtotal or total), as meniscus being a very important shock absorber present between the knee joint can lead to delay in osteoarthritis of knee joint. Therefore, our priority should be to save the 
meniscus and restore the normal anatomy of the knee joint for its biomechanical stability and normal function.

Funding: No funding sources

Conflict of interest: None declared

Ethical approval: The study was approved by the institutional ethics committee

\section{REFERENCES}

1. Walker PS, Hajek JV. The load-bearing area in the knee joint. Biomech. 1972;5:581-9.

2. Voloshin AS, Wosk J. Shock absorption of meniscectomized and painful knees: a comparative in vivo study. J Biomed Eng. 1983;5:157-61.

3. Mac CM. The movements of bones and joints; the synovial fluid and its assistants. J Bone Joint Surg Br. 1950;32:244-52.

4. Zimny ML, Albright DJ, Dabezies E. Mechanoreceptors in the human medial meniscus. Acta Anat (Basel). 1988;133:35-40.

5. BB Seedhom, Dowson D, Wright V. Proceedings: functions of the menisci. A preliminary study. Ann Rheum Dis. 1974;33:111.

6. Fairbank TJ. Knee joint changes after meniscectomy. J Bone Joint Surg Am. 1948;30B:664-70.

7. Baratz ME, Fu FH, Mengato R. Meniscal tears: the effect of meniscectomy and of repair on intraarticular contact areas and stress in the human knee. A preliminary report. Am J Sports Med. 1986;14:270-5.

8. Campbell SE, Sanders TG, Morrison WB. MR imaging of meniscal cysts: incidence, location, and clinical significance. AJR Am J Roentgenol. 2001;177:409-13.

9. Deveci A, Cankaya D, Yilmaz S, Özdemir G, Arslantaş E, Bozkurt M. The arthroscopical and radiological corelation of lever sign test for the diagnosis of anterior cruciate ligament rupture. Springerplus. 2015;4:830.

10. Choi NH, Kim TH, Victoroff BN. Comparison of arthroscopic medial meniscal suture repair techniques: inside-out versus all-inside repair. Am J Sports Med. 2009;37:2144-50.

11. Stein T, Mehling AP, Welsch F, von Eisenhart-Rothe $\mathrm{R}$, Jäger A. Long-term outcome after arthroscopic meniscal repair versus arthroscopic partial meniscectomy for traumatic meniscal tears. Am J Sports Med. 2010;38(8):1542-8.

12. Lutz C, Dalmay F, Ehkirch FP, Cucurulo T, Laporte C, Le Henaff G et al. French Arthroscopy Society. Meniscectomy versus meniscal repair: 10 years radiological and clinical results in vertical lesions in stable knee. Orthop Traumatol Surg Res. 2015;101(8):S327-31.

13. Barrett GR, Treacy SH, Ruff CG. Preliminary results of the T-fix endoscopic meniscus repair technique in an anterior cruciate ligament reconstruction population. Arthroscopy. 1997;13:218-23.

14. Kimura M, Shirakura K, Higuchi H, Kobayashi Y, Takagishi K. Eight to 14-year follow-up of arthroscopic meniscal repair. Clin Orthop Relat Res. 2004:175-80.

15. Kocabey Y, Nyland J, Isbell WM, Caborn DNM. Patient outcomes following T-Fix meniscal repair and a modifiable, progressive rehabilitation program, a retrospective study. Arch Orthop Trauma Surg. 2004;124:592-6.

16. Austin KS, Sherman OH. Complications of arthroscopic meniscal repair. Am J Sports Med. 1990;21:864-69.

17. Stone RG, Frewin PR, Gonzales S. Long-term assessment of arthroscopic meniscus repair: A two- to six-year follow-up study. Arthroscopy. 1990;6:73-8.

18. Albertoni LJB, Schumacher FC, Ventura MHA, Da Silveira Franciozi CE, Debieux P, Kubota MS et al. Meniscal repair by all-inside technique with Fast-Fix device. Rev Bras Ortop Engl Ed. 2013;48:448-54.

19. Haas AL, Schepsis AA, Hornstein J, Edgar CM. Meniscal repair using the Fast-Fix all-inside meniscal repair device. Arthroscopy. 2005;21(2):167-75.

20. Uzun E, Misir A, Kizkapan TB, Ozcamdalli M, Akkurt S, Guney A. Evaluation of Midterm Clinical and Radiographic Outcomes of Arthroscopically Repaired Vertical Longitudinal and Bucket-Handle Lateral Meniscal Tears. Orthop J Sports Med. 2019;7:5.

Cite this article as: Singh SP, Singh M, Singh PV, Singh S, Arif M. Functional outcome of arthroscopic meniscal repair in meniscal tears: a prospective study in tertiary care hospital in North India. Int J Res Orthop 2021;7:816-9. 